\title{
Gamma Rays Provide New Quantum Gravity Constraint
}

\author{
An analysis of the speed of the most energetic photons ever observed \\ from a gamma-ray burst sets new constraints on certain theories of \\ quantum gravity.
}

By Marric Stephens

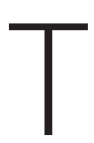

o develop a theory of quantum gravity-the holy grail of theoretical physics-researchers might need to sacrifice one of the field's central principles: the invariance of the speed of light. The predictions of general relativity and quantum theory are expected to coincide somewhere near the Planck energy (about $1.22 \times 10^{19} \mathrm{GeV}$ ) (see Focus: The Period of the Universe's Clock). But some theories indicate consequences at far lower energies, which would manifest as modifications to the propagation speed of very-high-energy gamma rays (VHEGRs). Now, by analyzing the arrival times of the most energetic photons ever measured from a gamma-ray burst (GRB 190114C), the MAGIC Collaboration shows that if the photons' speed does deviate from the speed of light, it is by a factor of less than $1.7 \times 10^{-15}$.

The best place to find evidence of photons traveling faster or slower than the speed of light is in signals that have traversed

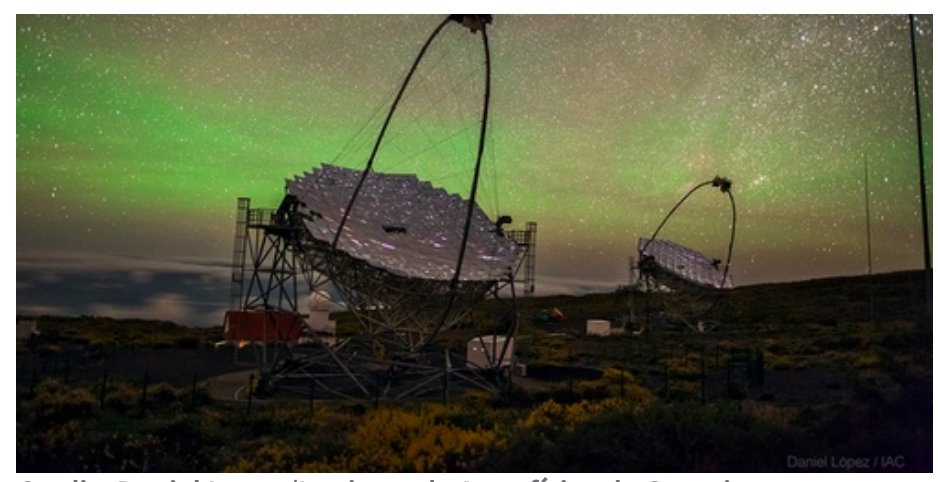

Credit: Daniel Lopez/Instituto de Astrofísica de Canarias much of the Universe. This colossal distance is needed to make detectable the tiny potential discrepancy in the speeds of photons of different energies. At 4.5 billion light years, GRB $190114 C$ was closer than other GRBs that have been used to constrain quantum gravity theories. But its photons were more energetic, coming in at $2 \mathrm{TeV}$, compared with less than $100 \mathrm{GeV}$ for previously measured events. This high energy allowed a unique test of a specific light-speed invariance-violation model in which the relationship between photon energy and velocity is quadratic.

The team found that the speed of photons from GRB $190114 \mathrm{C}$ matches the speed of light, indicating that under the invariance-violation model, unification must occur at an energy above $5.6 \times 10^{10} \mathrm{GeV}$. They hope that future observations of more distant GRBs will let them tighten the constraint.

This research is published in Physical Review Letters.

Marric Stephens is a Corresponding Editor for Physics based in Bristol, UK. 